Acta Technologica Agriculturae 2

Nitra, Slovaca Universitas Agriculturae Nitriae, 2020, pp. 73-80

\title{
APPLICATION OF PERCOLATION THEORY IN THERMOKINETICS
}

\author{
Alok DHAUNDIYAL ${ }^{1 *}$, Suraj BHAN SINGH ${ }^{2}$ \\ ${ }^{1}$ Szent István University, Gödöllő, Hungary \\ ${ }^{2}$ Govind Ballabh Pant University of Agriculture and Technology, Pantnagar, Uttarakhand, India
}

The present paper focuses on the infiltration of random fluid particles through a porous wall. The thermogravimetric curve is assumed to be a non-eroded interface so that the random fluid particle can pass through the discrete boundary of it. The node on the thermogravimetric plane is denoted by the three-dimensional lattice, which has unit thickness. The Lattice Boltzmann method is implemented to determine the variation of the remaining mass fraction $(X)$ with respect to time. The kinetic model is correlated with the Lattice Boltzmann method to determine the kinetic parameters. The practical situation of Moka coffee maker is applied to determining the kinetic parameters.

Keywords: kinetic model; Lattice Boltzmann method; percolation; kinetic parameters; thermogravimetry

This paper is based on the assumptions that the discontinuity of a curve can be treated as a passage for a fluid element to pass out of the three-dimensional thermogravimetric plane and the rate of change of displacement corresponds to the variation of remaining mass fraction. The displacement of a fluid element with respect to the time can be represented through a mass distribution function associated with velocity and flow density. In this article, a physical model of percolation of coffee is correlated with the chemical kinetic model. Temperature and time are assumed to be related to the three-dimensional node of a fluid particle. Percolation concept comprises two stages: ingression of fluid elements through the inlet and passing through a porous medium with the help of body force or a low-pressure gradient. Furthermore, it wets the porous medium and expels the air. The body force or existence of a low-pressure gradient makes the small particles of porous interface detach from larger grains, and they drain away with the fluid. As a result of erosion, they can either deposit on other grains, or get sedimented. A Lattice Boltzmann method (LBM) is implemented to determine the mass flow rate of the hypothetical fluid element through the porous plane. The basic Lattice Boltzmann D3Q19 (three-dimensional and 19 velocities) (Habich et al., 2011) model is used to simulate percolation of the fluid elements across the plane. The boundary of the porous wall is assumed to be non-eroded, which means it will not dissolve or lose sub-particle in the hypothetical fluid. The broad perspective of LBM covers the simulation of fluid flow. It solves the Boltzmann equation for a discrete lattice and assists in recovering of the NavierStrokes equation by reducing the Mach number. Although the LBM has compressibility effect, it can be reduced to the incompressible fluid if dilatational viscosity is negligible.
The method can be applied to the simulation of flow in porous media (Kutay et al., 2006), colloidal suspensions (Harting et al., 2008), liquid-gas phase transitions, multicomponent flow (Huang et al., 2011; Nekovee et al., 2000), and spinodal decomposition (Giupponi et al., 2006). Besides the microscopic application, the LBM may have a wide scope in the macroscopic issues of gasification and pyrolysis. The paper focuses on the implementation of percolation theory to simulate the remaining mass fraction of hardwood and correlate it with a multi-reaction model in order to evaluate the relevant kinetic parameters with the help of the inverse solution. Unlike the Monte Carlo method (Dhaundiyal et al., 2019), the concept of percolation is based on the dynamic nature of fluid nodes. Overlapping of random fluid particles and nonhomogeneous concentration of fluid and void space impart a significant error while measuring the area bounded by the TG curves. Therefore, it must be tackled in such a manner so that the error encountered whilst evaluating kinetic parameters can be minimised.

\section{Material and methods}

\section{The Lattice Boltzmann method for fluid simulation}

The LBM is used to solve the Boltzmann equation (Eq. 1) for a discrete system. It defines the dynamic behaviour of a gas in a microscopic system. The collision of gas particles with velocity $v_{i}$ is highly stochastic and, hence, it exchanges momentum among themselves. The total momentum and energy of the system are conserved for the ideal collision. Role of the Boltzmann equation is to determine the velocity of a particle as a probability function of position and time, $f(k, t)$. 


$$
v \cdot \nabla_{k} f+F \cdot \nabla_{p} f+\frac{d f}{d t}=\Omega(f(k, t))
$$

where:

$f \quad$ - external body force or mass distribution function

$\nabla_{k}, \nabla_{p} \quad-$ gradient in position and momentum space respectively

$\Omega(f) \quad$ - collision operator

The collision operation relationship (Eq. 2) with the equilibrium distribution and relaxation time $\tau$ is proposed by Bhatnagar, Gross and Krook (BGK dynamics) (Bhatnagar et al., 1954).

$$
\hat{\Omega}(f)=-\left(\frac{f_{i}-f_{i}^{e q}}{\tau}\right)
$$

Here, the equilibrium value $f^{e q}$ is obtained by discretising the Boltzmann distribution (Eq. 3). The expression is limited up to the second order (Li et al., 2013; Guo et al., 2006).

$$
f_{i}^{e q}(\rho, v)=w_{i} \rho\left[1+\frac{c_{i} v}{\left(c_{s}\right)^{2}}+\left(\frac{c_{i} v}{\left(c_{s}\right)^{2}}\right)^{2}-\frac{v^{2}}{2 c_{s}^{2}}\right]
$$

where $w_{i}=\left\{\begin{array}{l}\frac{1}{3}, i=0 \\ \frac{1}{18}, i=1 . ., 6 \\ \frac{1}{36}, i=7,8 \ldots, 18\end{array}\right.$ denotes the lattice weights, and $c_{s}=\frac{1}{\sqrt{3}}$ is the lattice speed of sound for D3Q19. The equilibrium distribution function for a fluid model depends on the local density $\rho(k, t)$ and the velocity field $v(k, t)$. The LBM discretises the probability density function $f$ in space and time. The equilibrium distribution $f^{e q}$ depends on the lattice type used for the fluid simulation. But the most popular and widely used for 3D lattice fluid simulation is D3Q19 (Hecht and Harting, 2010), where each node has 19 links. The other three-dimensional lattice models used in fluid simulation are D3Q15 and D3Q27. The D3Q27 has the highest computational effort, whereas the D3Q15 has the least stability among the 3D models. Apart from 3D lattice models, for 2D simulation, D2Q9, which consists of 9 discrete velocity directions, is used for two-dimensional fluid flow. For each lattice site, 19 distribution functions $\left(f_{i}(k, t)\right)$ are used. Each lattice has assigned a lattice velocity $C_{i}$ vector. The subscript $i$ denotes the $i^{\text {th }}$ column of lattice matrix $J$. The six velocity directions along the cartesian co-ordinate and the one stationary velocity at the centre is expressed in Eq. 4.

$J=\left[\begin{array}{rrrrrrrrrrrrrrrrrrr}1 & -1 & 0 & 0 & 0 & 0 & 1 & 1 & 1 & 1 & -1 & -1 & -1 & -1 & 0 & 0 & 0 & 0 & 0 \\ 0 & 0 & 1 & -1 & 0 & 0 & 1 & -1 & 0 & 0 & 1 & -1 & 0 & 0 & 1 & 1 & -1 & -1 & 0 \\ 0 & 0 & 0 & 0 & 1 & -1 & 0 & 0 & 4 & -1 & 0 & 0 & 1 & -1 & 1 & -1 & 1 & -1 & 0\end{array}\right]$ (4)

The geometry of 3D lattice D3Q19 is illustrated in Fig.1. The local density (Eq. 5) at a lattice point can be derived by summing the mass distribution function $f$ :

$$
\rho(k, t)=\sum_{i=0}^{18} f_{i}(k, t)
$$

The local streaming velocity $v(k, t)$ can be obtained from the following expression (Eq. 6):

$$
v(k, t)=\frac{\sum_{i=0}^{18} C_{i} \cdot f_{i}(k, t)}{\rho(k, t)}
$$

The single-particle distribution for any lattice model at lattice node $k$ is given by the Boltzmann equation (Eq. 7) for time evolving mass distribution function $f(k, t)$ :

$$
f\left(k+C_{i}, t+1\right)=f_{i}(k, t)+\Omega(f(k, t))
$$

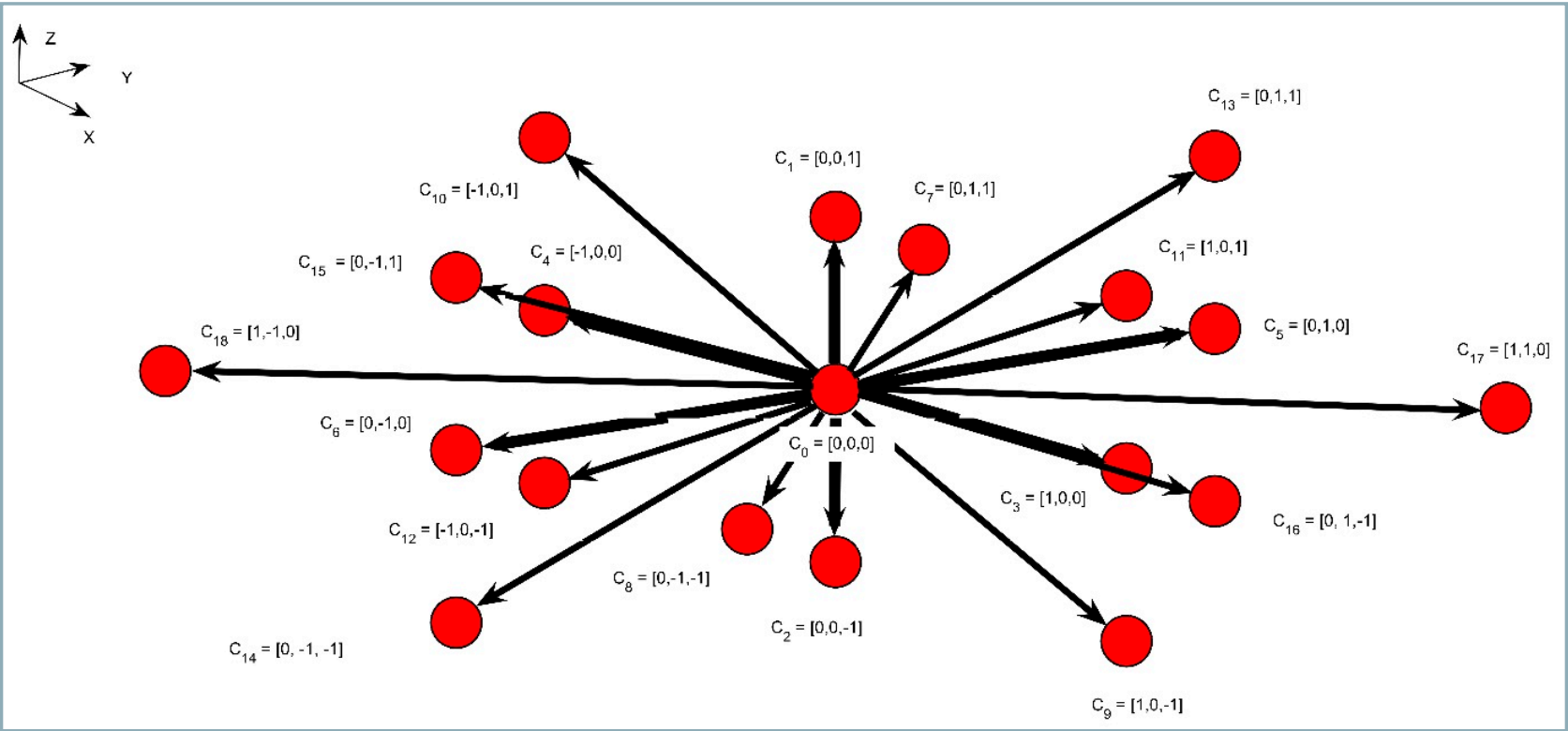

Fig. 1 Geometry of the D3Q19 with distinct lattice velocities $\left(C_{i}, i=0,1,2 \ldots .18\right)$ 
There are also several assumptions related to the LBM that must be known before applying it to the macroscopic scale. Some of them are: external forces are neglected; movement of the particles take place only along the lattice vectors; the single relaxation time approximation for BGK; modelling of the fluid by numerous cells having the same type; updating the cells at each time step; and storage of the number of particles that move along each of the lattice vector particle distribution functions. However, there are also MRT (multiple-relaxation time) approximations. For the given issue, the air trapped at discontinuity must be displaced by a hypothetical fluid node so that the fluid flow properties can be correlated to the rate of change of the remaining mass fraction, which, in a physical system, is a vertical height of the TG plane. Such a system transforms the issue into two-fluid model. In the current scenario, the orange balls represent the fluid elements and the white dots on the TG plane represent the air void (Fig. 6). It is assumed that the plane is non-fluidizable, thus the behaviour of fluid along the plane predicts the same behaviour as the remaining mass fraction. The indicator function $\zeta$ is used to determine whether air void is replaced by a fluid node or not.

$$
\zeta(k)=\left\{\begin{array}{r}
1, \mid \beta(k, t) \geq 1 \\
0, \mid \beta(k, t) \leq 0 \\
0.5(\sin (\pi(\beta(k, t)-0.5))+1), 0<\beta(k, t)<1
\end{array}\right.
$$

Here $\beta(k, t)=\sum_{i=0}^{18} \beta_{i}(k, t)$.

The indicator function $\zeta(k)=1$ if the fluid node replaces the air void, else $\zeta(k)=0$. On the TG plane boundary $(0<\beta(k, t)<1)$, the indicator function has the sinusoidal behaviour (Eq. 8). So, similarly, for air void, the equation of collision step can be represented by Eq. (9):

$$
\beta\left(k+C_{i}, t+1\right)+\eta_{\beta}\left(\beta_{i}(k, t)-\beta_{i}^{e q}(k, t)\right)=\beta_{i}(k, t)
$$

where:

$\eta_{\beta}$ - relaxation parameter

$\beta_{i}^{\text {eq }}(k, t)$ - equilibrium function, which can be given by the following expression:

$$
\beta_{i}^{e q}(k, t)=\frac{\beta_{i}(k, t) f_{i}(k, t)}{\rho}
$$

The parameter $\eta(k, t)$ helps in determining diffusivity of momentum between various layers of fluid and at each time step, it depends upon the value of indicator function $\zeta(k)$ :

$$
\eta(k, t)=\zeta(k) \eta_{f}(k, t)+(1-\zeta(k)) \eta_{a}(k, t)
$$

where:

$\eta_{f}, \eta_{a}-$ relaxation parameters for hypothetical fluid and air respectively

The rate of change of mass fraction of fluid along the plane is:

$$
\frac{d x}{d t}=\frac{\int_{s}[\rho(k, t) v(k, t) \hat{n}] d s}{1,642,346.626 \rho_{f}} \simeq \frac{\sum_{k=1}^{N} \rho(k, t) v(k, t) \delta s}{1,642,346.626 \rho_{f}}
$$

where:

$\hat{n}-$ unit vector normal to the surface area of TG plane

$N$ - number of fluid particles settle on the TG plane

Integrating Eq. 12 with respect to ' $t$ ', we have $X_{f} \simeq \frac{\left(\sum_{k=1}^{N} \rho(k, t) v(k, t) \delta s\right) \delta t}{1,642,346.626 \rho_{f}}\left(X_{f}-\right.$ mass fraction of fluid on the plane).

$$
\begin{aligned}
& \text { Here, } v=\left(v_{x}^{2}+v_{y}^{2}+v_{z}^{2}\right)^{\frac{1}{2}} \\
& \begin{aligned}
\rho v_{x} & =f_{1}+f_{7}+f_{8}+f_{9}+f_{10}-\left(f_{2}+f_{11}+f_{12}+f_{13}+f_{14}\right) \\
\rho v_{y} & =f_{3}+f_{7}+f_{11}+f_{15}+f_{16}-\left(f_{4}+f_{8}+f_{12}+f_{17}+f_{18}\right) \\
\rho v_{z} & =f_{5}+f_{9}+f_{13}+f_{15}+f_{17}-\left(f_{6}+f_{10}+f_{14}+f_{16}+f_{18}\right)
\end{aligned}
\end{aligned}
$$

\section{Multi-reaction model for hardwood pyrolysis}

For correlating of the fluid flow with the thermogravimetric behaviour of hardwood, an interface is required to derive the relevant parameters related to the pyrolysis of biomass. Distributed Activation Energy Model (DAEM) is adopted to find the inverse solution of the given issue. To obtain a realistic solution, it is necessary to know the parameters that affect the model solution. Comprehensive information on various parameters of the non-isothermal $n^{\text {th }}$ order is reported by Brown (1988). The basic assumptions and their derivations can be found in Dhaundiyal and Tewari (2017); Dhaundiyal and Singh (2019); Burnham (2017). The nonisothermal $n^{\text {th }}$ DAEM are as follow:

the first order reaction:

$$
X=\int_{0}^{\infty} \exp \left(\int_{0}^{t}-A \exp \left(-\frac{E}{R T}\right) d t\right) f(E) d E
$$

the $n^{\text {th }}$ order:

$$
X_{n}=\int_{0}^{\infty}\left\{1-(1-n) \int_{0}^{t} A \exp \left(-\frac{E}{R T}\right) d t\right\}^{\left(\frac{1}{1-n}\right)} f(E) d E
$$

where:

$E \quad$ - activation energy

A - pre-exponential factor

$R \quad$ - ideal gas constant

$n$ - reaction order

$t$ - time

$X$ - remaining mass fraction of releasing volatiles

$f(E)$ - density function of activation energies, which is usually represented by the stochastic models

Many stochastic models have been used to determine the relevant kinetic parameters, but the most reliable model is Rayleigh distribution and Gaussian distribution (Dhaundiyal and Tewari, 2017; Dhaundiyal and Singh, 2020; Dhaundiyal and Toth, 2020). The redundant distribution parameters cause over-fitting of model, which is difficult to interpret within the desirous range; therefore, the study is focussed on the simplest form of density function, i.e. the Gaussian distribution, which was primarily used in coal 
pyrolysis (Serio et al., 1987) and later became very popular in the DAEM for modelling thermochemical processes (Galgano and Blasi, 2003). The Eqs. 12 and 13 have no analytical solution; therefore, it is required to approximate the double integral form of remaining mass fraction variation with time and temperature. The Laplace integral method (Dhaundiyal et al., 2019; Dhaundiyal and Toth, 2019) is used to obtain the approximation for double exponential term and double integral form. The basic notion behind using the Laplace integral method is to transform both the functions into a single dimensionless variable so that it becomes easier to tackle the multi-variable problem. To approximate multi-reaction model, the activation energy is rescaled by using the scaling factor $y$, whereas the corresponding energy correction factor (y) for the double exponential term is denoted by $y_{s}$ and $y_{w}$. The subscript 's' and ' $w$ ' denotes the mean and step width of the activation energy. It implies the activation energy varies around mean values with step size of $y_{w}$.

$$
y=\frac{E}{E_{0}}
$$

where:

$E_{0} \quad$ - the mean value of activation energy

Eq. 16 can be rewritten as a combination of two functions:

$$
X=\int_{0}^{\infty} H(T, t) \cdot f(y) d y
$$

where:

$H(T, t)=\exp \left(\int_{0}^{t}-A \exp \left(-\frac{E}{R T}\right) d t\right)-$ double exponential term $f(y)=\frac{1}{E_{0}} \sqrt{\frac{\psi}{\pi}} \exp (\psi(y-1))^{2}-$ Gaussian distribution function of scale factor ' $y^{\prime} \cdot \psi=\frac{E_{0}^{2}}{2 \sigma^{2}}$ is the constant parameter $\sigma^{2}$ - variance of the distribution function

The function $H(T, t)$ can be approximated as:

$$
H(T, t)=\exp \left(\frac{-A R T t}{E} \exp \left(\frac{-E}{R T}\right)\right) \text { as } \frac{E}{R} \rightarrow \infty
$$

The exponent of exponential term $\left(\frac{-A R T t}{E} \exp \left(\frac{-E}{R T}\right)\right)$ exp in Eq. 13 can be rewritten as:

$$
H(T, t)=\exp \left(-\exp \left(\frac{E_{s}-E}{E_{w}}\right)\right)
$$

Since the exponent of exponential term behaves like a step function where $E$ varies around the mean value $E_{s}$ with step size of $E_{w}$, it is necessary to determine the behaviour of $E$ around neighbourhood of $E_{s}$. The significance of double exponential function $H(T, t)$ can be understood by varying the value of $E$. The function is nearly one for all the values $E$ greater than $E_{s}$, whereas the function approaches zero for the values of $E$ lower than $E_{s}$. In other words, the contribution of double exponential term in the DAEM can be known only if the boundaries of activation energy domain are defined.
Let $S(E)=\left(\frac{E_{s}-E}{E_{w}}\right)$.

Apply Taylor series expansion on $S(E)$ around $E=E_{S}$.

$$
S(E)=S\left(E_{s}\right)+\left(E-E_{s}\right) S^{\prime}\left(E_{s}\right)+\ldots
$$

The boundary conditions at $E=E_{s}$ are $S\left(E_{s}\right)=0$ and $S^{\prime}\left(E_{s}\right)=-\frac{1}{E_{w}}$.

After solving Eqs. 18; 19; and 20, we get:

$$
E_{s}=R T \cdot W(A \cdot t) \text { and } E_{w}=\frac{E_{s}}{E_{s}+R T}
$$

where:

$W(A \cdot t)$ - the Lambert $W$ function

Thus, the Eq. 16 becomes:

$$
X=\int_{0}^{\infty} \exp \left(-\exp \left(\frac{y_{s}-y}{y_{w}}\right)\right) \cdot \sqrt{\frac{\psi}{\pi}} \exp (\psi(y-1))^{2} d y
$$

Applying Laplace asymptotic method to Eq. 21, we will get:

$$
x_{n=1} \sim \frac{\exp \left(-\psi\left(y_{e}-1\right)\left(y_{e}+2 y_{w}-1\right)\right)}{\sqrt{1+\left(\frac{y_{e}-1}{y_{w}}\right)}}
$$

where:

$y_{e}$ - location where the integrand (Eq. 21) has a maximum value:

$$
y_{e}=1+y_{w} \cdot W\left(\frac{1}{2 \psi y_{w}^{2}} \exp \left(\frac{y_{s}-1}{y_{w}}\right)\right)
$$

Similarly, for the $n^{\text {th }}$ order, we have:

$$
\begin{aligned}
X_{n \neq 1} \sim 1 & -\frac{1}{\sqrt{2 \psi}}\left[\exp \left(-y\left(y_{e, n}-1\right)\left(2 y_{s}-y_{e, n}-1\right)\right)+\right. \\
+ & \left.\frac{n}{2} \exp \left(-y\left(y_{e, n}-1\right)\left(2 y_{s}-y_{e, n}-1\right)\right)\right]
\end{aligned}
$$

where:

$y_{e, n}=1-\frac{1}{2 \psi y_{w}}$

Eqs. 22 and 23 are the required expressions.

\section{Experimental setup}

A pilot size reactor has been used to test the hardwood chip (G50). The raw material, hardwood (Acacia), has been gathered in Pest county of Hungary. The processed raw material has been obtained by shredding the billet of wood. The properties of thermodynamic system - pressure and temperature - have been measured using pressure and temperature sensors placed inside the main chamber. Temperature sensor has been located $80 \mathrm{~mm}$ from the 
Table 1 Ultimate analysis of hardwood (Acacia) (dry basis)

\begin{tabular}{|c|c|c|c|c|c|c|c|c|}
\hline $\mathrm{C}(\%)$ & H (\%) & N (\%) & $\mathrm{O}(\%)$ & S (\%) & Ash (\%) & $\mathrm{Cl}(\%)$ & Energy density (GJ.m $\left.{ }^{-3}\right)$ & N.C.V (MJ.kg $\left.{ }^{-1}\right)$ \\
\hline 50.03 & 5.849 & 0.075 & 42.947 & 0.061 & 1.037 & 0.02 & 10.88 & 18.70 \\
\hline
\end{tabular}

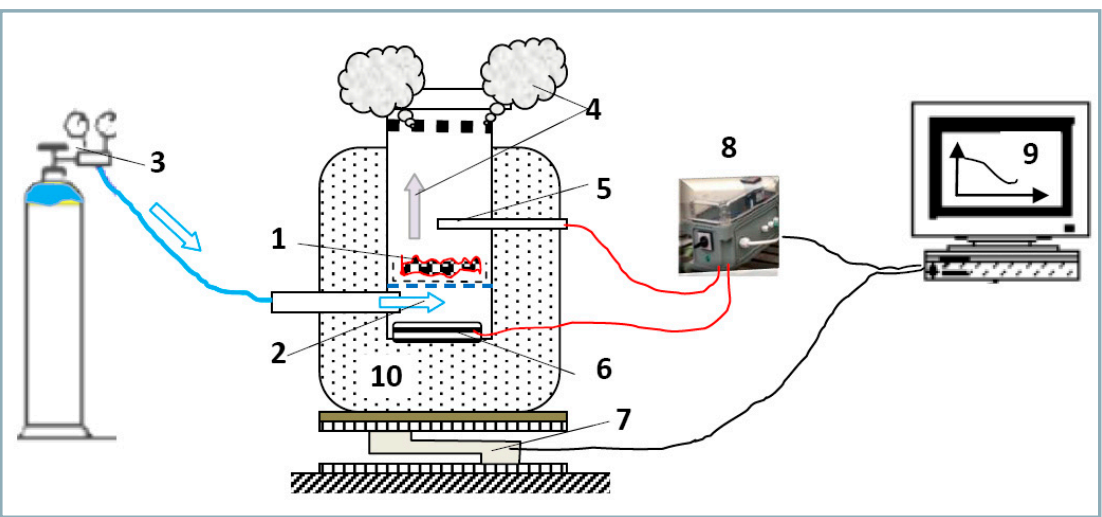

Fig. 2 Schematic diagram of the pyrolysis unit

1 - grate; 2 -nozzle; 3 - regulating valve; 4 - pyrolysis gas; 5 -temperature sensor (Nickel-Chromium/Nickel-Alumel); 6 - pressure sensor; 7 - weight sensor; 8 - data logger; 9 - processing unit; 10 - mineral wool insulator reactor base, while the pressure sensor has been at reactor base. The inert gas, nitrogen, has been allowed to flow at volumetric rate of $70 \mathrm{~mL} \cdot \mathrm{s}^{-1}$. The ' $K$ ' type ((Nickel-Chromium/NickelAlumel) thermocouple has been used to measure the sample temperature. The weight sensor has been placed beneath the heating chamber separated from the mineral wool covering. The indirect heating has been provided by a heating element of 2 kWe (Hertz, Germany). The grate has been made up of $0.7 \mathrm{~mm}$ stainless steel, whereas the heat resisting material - rock wool - has been used to separate reactor from the outer casing. Reactor core has been made of $1.5 \mathrm{~mm}$ welded carbon steel. The inner reactor diameter has been $110 \mathrm{~mm}$; the reactor surface diameter - 210 $\mathrm{mm}$. The schematic diagram of pilot size pyrolysis unit is illustrated in Fig. 2. Elemental composition of the raw material has been measured by $\mathrm{CHNS}$ analyser (vario MACRO cube, Germany). The calorific value of hardwood has been obtained using the bomb calorimeter at constant volume. The ultimate analysis of hardwood (Acacia) is shown in Table 1.

\section{Results and discussion}

To calculate the kinetic parameters of biomass pyrolysis, the concept of percolation is used. The process is exactly the same as making the Moka coffee in a Moka pot, except in place of kettle, a mathematical plane has been used as a perforated filter to

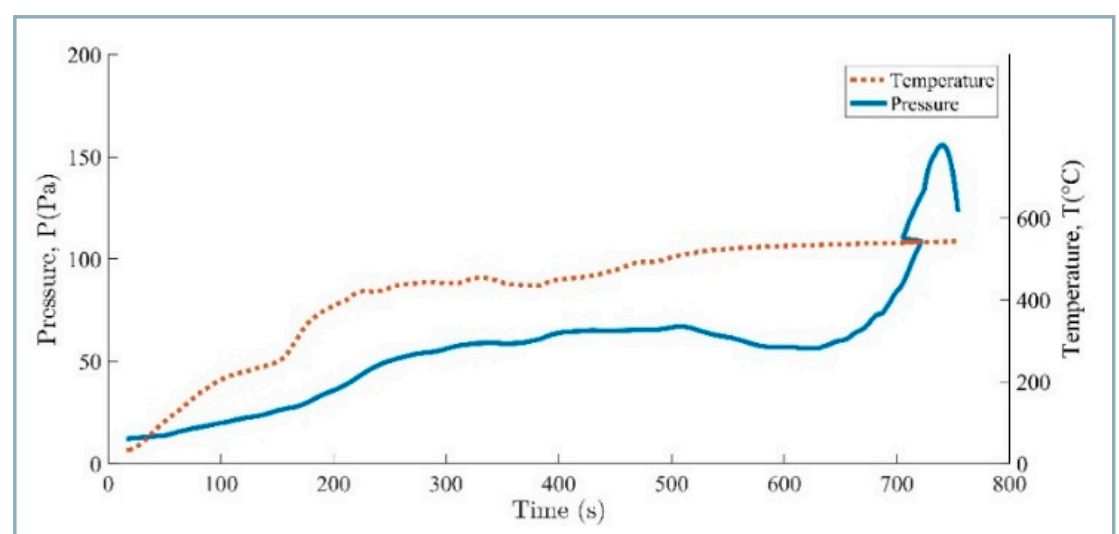

Fig. 3 Temperature and pressure variation across the pyrolysis reactor allow the hypothetical fluid to seep through the discreet boundaries. The plane used for demonstration is the thermogravimetric plane (TG), which is obtained by pyrolysis of G50 hardwood (Acacia) chips in a pilot-size reactor.

In order to correlate this physical event to the chemical process, the principle of lattice gas is used. The LBM is used as a mathematical interface to introduce the kinematics of fluid element. The D3Q19 (three-dimensional model with 19 lattice velocities) has been adopted to understand the mesoscopic process. To simplify the calculation, the boundaries are not fluidised with flow; therefore, the biomass nodes are intact throughout the process. The fraction of fluid on the outer surface of TG thermogram has a common mathematical solution, as it is obtained through multi-reaction model. In other words, the rate of displacement of fluid element is proportional to the rate of change of biomass fraction in a pyrolysis reactor. Since the volatile and water content flows through the porous wood chips, the physical phenomenon of fluid flow can be correlated to the chemical kinetics. The temperature and pressure variation at the bed are illustrated in Fig. 3. Both system properties vary exponentially, except pressure shows a sudden jump due to changing regime of the fixed bed with respect to superficial velocity of pyrolysis gas. The thermogravimetric behaviour of hardwood chip is illustrated in Fig. 4a. Certain notches or small peaks, which are more perceptible in TGA analyser, in the TG curve obtained from Industrial reactor (Fig. $4 \mathrm{~b}$ and Fig. 9) show decomposition stages of hardwood. The fluid elements are generated through the MATLAB and the volume enclosed by the TG plane (Fig. 4b) is obtained with the help of the Monte-Carlo method. The numerical solution of the prosed LBM is determined by using 
Table 2 Comparison of kinetic parameters obtained from different methodologies

\begin{tabular}{|c|c|c|c|c|c|}
\hline \multirow[t]{2}{*}{ Parameters } & \multicolumn{2}{|c|}{ LBM } & \multirow{2}{*}{$\begin{array}{c}\text { Monte Carlo } \\
\text { (Dhaundiyal et al., 2019) }\end{array}$} & \multirow{2}{*}{$\begin{array}{c}\text { Model-free } \\
\text { (Wilk et al., 2017) }\end{array}$} & \multirow{2}{*}{$\begin{array}{l}\text { Model-fitting } \\
\text { (Oluoti et al., 2014) }\end{array}$} \\
\hline & $n=1\left(R^{2}=0.98\right)$ & $n=2\left(R^{2}=0.33\right)$ & & & \\
\hline $\bar{E}$ & $190.49 \mathrm{~kJ} \cdot \mathrm{mol}^{-1}$ & $7.24 \mathrm{~kJ} \cdot \mathrm{mol}^{-1}$ & $35.92 \mathrm{~kJ} \cdot \mathrm{mol}^{-1}$ & $180 \mathrm{~kJ} \cdot \mathrm{mol}^{-1}$ & $95.41 \mathrm{~kJ} \cdot \mathrm{mol}^{-1}$ \\
\hline $\bar{A}$ & $1.93 \times 10^{41} \mathrm{~min}^{-1}$ & $8.1 \times 10^{25} \mathrm{M}^{-1} \cdot \mathrm{min}^{-1}$ & $1.06 \times 10^{4} \mathrm{~min}^{-1}$ & $1.15 \times 10^{11} \mathrm{~min}^{-1}$ & $3.20 \times 10^{12} \mathrm{~min}^{-1}$ \\
\hline$E_{\min }$ & $8.44 \mathrm{~kJ} \cdot \mathrm{mol}^{-1}$ & $4.22 \mathrm{~kJ} \cdot \mathrm{mol}^{-1}$ & $15 \mathrm{~kJ} \cdot \mathrm{mol}^{-1}$ & $125.29 \mathrm{~kJ} \cdot \mathrm{mol}^{-1}$ & $79.23 \mathrm{~kJ} \cdot \mathrm{mol}^{-1}$ \\
\hline$E_{\max }$ & $967 \mathrm{~kJ} \cdot \mathrm{mol}^{-1}$ & $63.24 \mathrm{~kJ} \cdot \mathrm{mol}^{-1}$ & $60 \mathrm{~kJ} \cdot \mathrm{mol}^{-1}$ & $226.75 \mathrm{~kJ} \cdot \mathrm{mol}^{-1}$ & $142.87 \mathrm{~kJ} \cdot \mathrm{mol}^{-1}$ \\
\hline
\end{tabular}

Table 3 Modelling parameters related to thermokinetics of Hardwood pyrolysis

\begin{tabular}{|l|c|c|}
\hline Parameter & First order & Second order \\
\hline $\boldsymbol{E}_{\boldsymbol{s}}$ & $0.24-492.92 \mathrm{~kJ} \cdot \mathrm{mol}^{-1}$ & $2.40-61 \mathrm{~kJ} \cdot \mathrm{mol}^{-1}$ \\
\hline $\boldsymbol{E}_{w}$ & $0.13-3.75 \mathrm{~kJ} \cdot \mathrm{mol}^{-1}$ & $1.44-6.1 \mathrm{~kJ} \cdot \mathrm{mol}^{-1}$ \\
\hline $\boldsymbol{A}$ & $0.00375-4.4 \times 10^{57} \mathrm{~min}^{-1}$ & $0.01086-5.43 \times 10^{26} \mathrm{M}^{-1} \cdot \mathrm{min}^{-1}$ \\
\hline
\end{tabular}
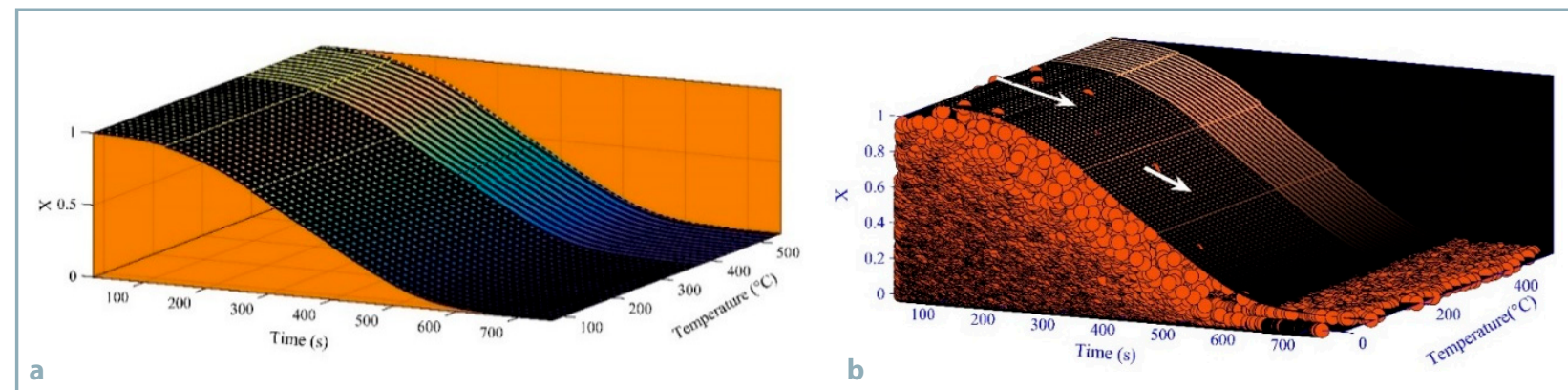

Fig. 4 (a) Thermogravimetric (TG) plane for the G50 wood chip; (b) Percolation of fluid element across the thermogravimetric (TG) plane

the iterative method. The distribution functions are obtained for each node (i) and its summation provided the mass density of fluid element at each node. The velocity of fluid is derived with sum of product of distribution function and the lattice velocities. On the basis of obtained solution, the mass fraction of fluid element on the surface is shown in Fig. 5. The fraction of generated fluid passed through plane is found to be $91 \%$ of total generated fluid element. The resultant distribution is negatively skewed, inferring the majority of mass density of fluid element gets shifted

to the higher temperature regime. The velocity field in terms of orthographic view at different time scale is illustrated in Fig. 6. It shows that the volumetric influx of fluid element is monumentally high at onset of simulation ( $t=2 \mathrm{~s})$. As time proceeds, the ingression of fluid element through porous medium gets subdued. The white porosity indicates discontinuity of plane, which is preoccupied with air. The air is assumed to be stagnant, thus its effect on simulation is absent. The governing equation for remaining mass fraction of biomass is represented by

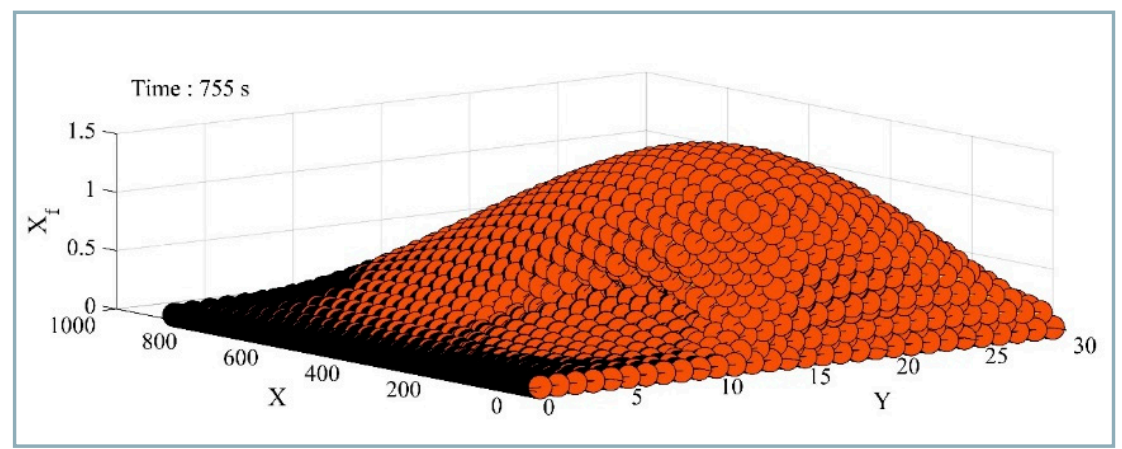

Fig. 5 Distribution of mass fraction (Xf) on the TG surface

distributed activation energy model. The relevant parameters related to biomass pyrolysis are derived by inverse formulation $(g(\theta, E, A)=$ $\left.f^{-1}\left(X_{n}\right)\right)$. The heating rate is found to be $53.78{ }^{\circ} \mathrm{C} \cdot \mathrm{min}^{-1}$, whereas the pre-exponential factor varies from $0.00375 \mathrm{~min}^{-1}$ to $4.4 e+57 \mathrm{~min}^{-1}$ for the first order reaction. The activation energy varies from $8.44 \mathrm{~kJ} \cdot \mathrm{mol}^{-1}$ to $947 \mathrm{~kJ} \cdot \mathrm{mol}^{-1}$. On the other hand, its average value is estimated to $190.49 \mathrm{~kJ} \cdot \mathrm{mol}^{-1}$. The results for the first order reactions are compared with the data obtained for same kind of hardwood (Dhaundiyal et al., 2019; Wilk et al., 2017; Oluoti et al., 2014) and it is found that the variation of activation energies, derived through model-free and modelfitting methodologies, fall within the range of solution estimated by the proposed scheme. To verify the potential existence of other solutions, the scheme is applied to higher order reactions, and the range of solution for activation energy - $E\left(\mathrm{~kJ} \cdot \mathrm{mol}^{-1}\right)$ - lies in the closed interval of $4.32 \leq E \leq 63.24$, 


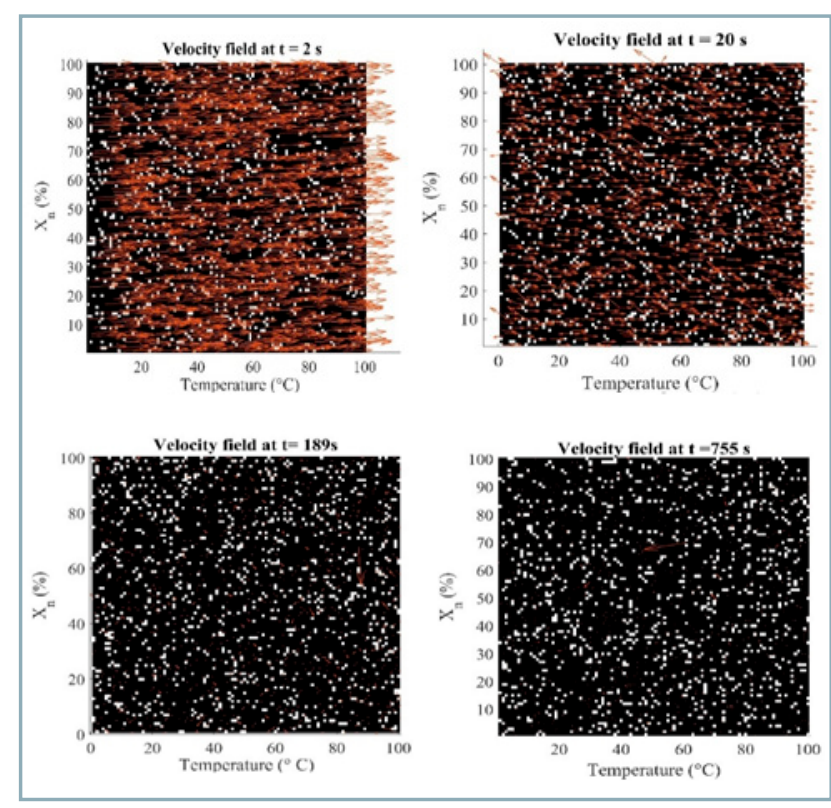

Fig. 6 Orthogonal projection of TG plane on the Y-Z plane (temperature scale: 1 scale unit $=5.4316^{\circ} \mathrm{C}$ )

whereas the obtained average value is $7.322 \mathrm{~kJ} \cdot \mathrm{mol}^{-1}$. The detailed parametric information is provided in Tables 2 and 3 . In the DAEM, the double exponential term is approximated by using stepwise variation of activation energy with the step size of $E_{w}$. The range of central value $\left(E_{s}\right)$, around which $E$ varies, falls in the closed interval of $0.24-492.92 \mathrm{~kJ} \cdot \mathrm{mol}^{-1}$, whereas the step-width $\left(E_{w}\right)$ varies from 0.13 to $3.75 \mathrm{~kJ} \cdot \mathrm{mol}^{-1}$. To understand the relative significance of double exponential term with respect to distribution function of activation energy $(f(E))$, the graphical plot is

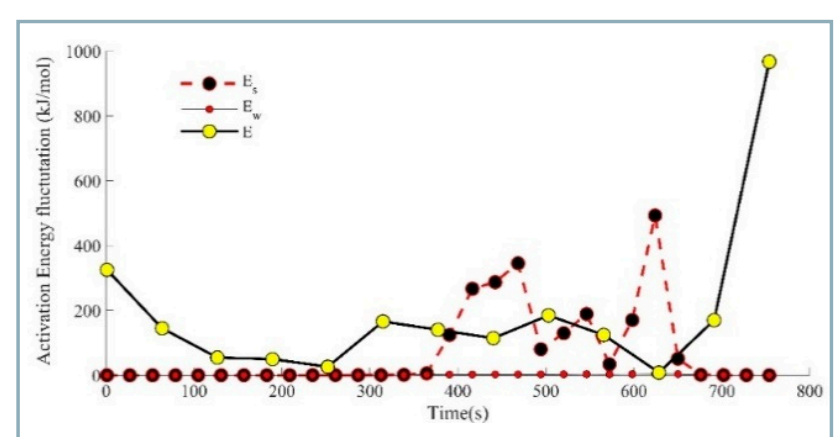

Fig. 7 Activation energy $\left(E, E_{s}, E_{w}\right)$ variation with respect to time

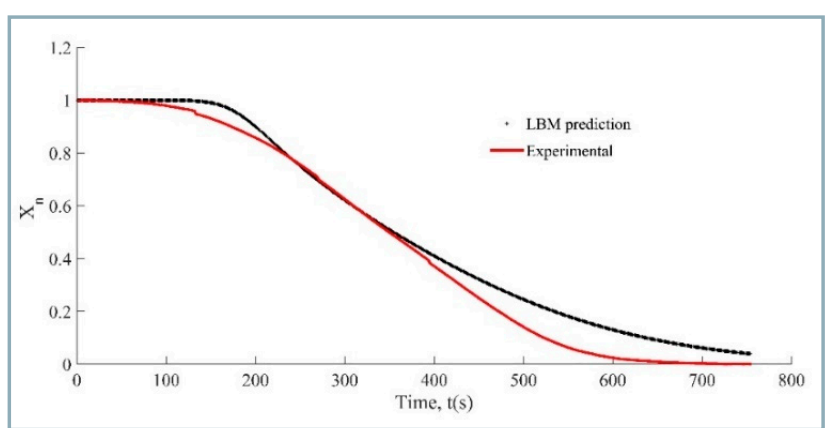

Fig. 9 Comparative sketch of predicted solution of the LBM $(n=1)$ and experimental result

drawn between activation energies and time (Fig. 7). The double exponential term has no significant role at onset of pyrolysis process. It merely affects the devolatisation and char formation of biomass, whereas the attribute of massloss curve of biomass at the beginning is guided by density function of activation energy. Therefore, it can be concluded that the double exponential function plays a vital role in thermokinetics, when the activation energy reaches its local minima in its interval. It can be more clearly understood by the surface plot (Fig. 8a) that the surface gradient of $E$ is relatively high in the beginning, whereas, with time, it decreases until it reaches the lowest point. Simultaneously, the temperature integral or double exponential function has its maximum gradient. Unlike the first order reaction, higher order reactions are predominately governed by the rate constant. An illustrative plot of activation energy variation across the plane is shown in Fig. 7b. It shows that the integrand is guided by the rate constant $\left(A \exp \left(-\frac{E}{R T}\right)\right)$ throughout the process, except at the beginning of the thermochemical conversion. The central value $E_{s}$ varies from 13 to $61 \mathrm{~kJ} \cdot \mathrm{mol}^{-1}$, while the step size $E_{w}$ is in the range of $1.44-6.1 \mathrm{~kJ} \cdot \mathrm{mol}^{-1}$. Mathematically, as the activation energies exceed $E_{s}$ the temperature integral will approach its global maxima; whereas, for all the values of $E$ at neighbourhood of $E_{s}$, the temperature integral will vary between 0 and 1, but it is the other way round for higher order reactions. According to the thermokinetic behaviour, the rate constant depends on $\mathrm{T}$ and $E$ and it attains its maximum value in the temperature range of $250-280{ }^{\circ} \mathrm{C}$ for hardwood pyrolysis, implying the temperature integral function has its significant effect in the given range for the first order reaction. Furthermore, it attains its local maxima at

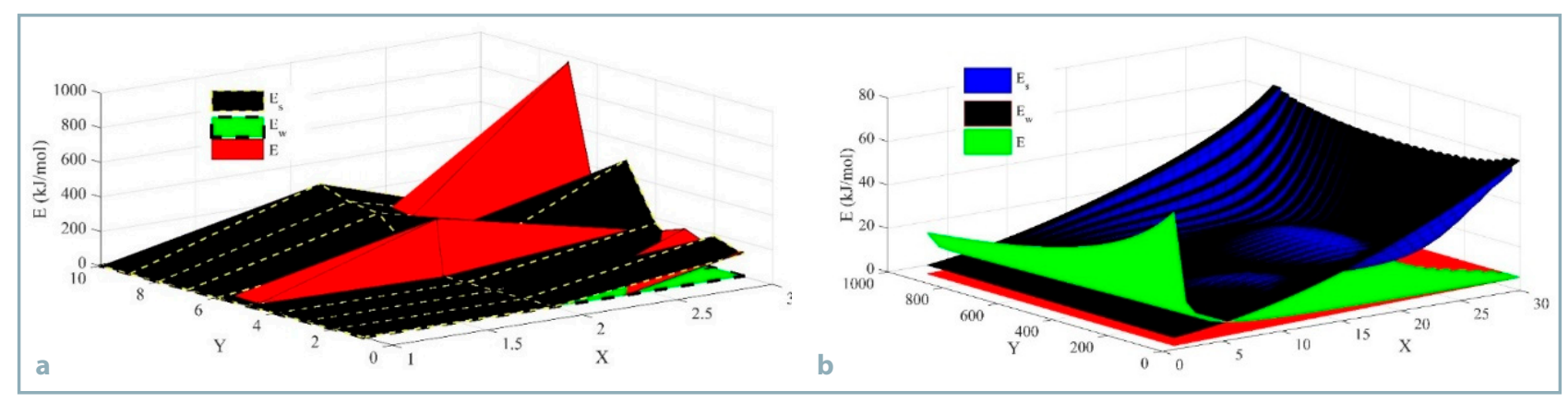

Fig. 8 (a) Relative variation of $E$ with respect to $E_{s}$ and $E_{w}(n=1)$; (b) Relative variation of $E$ with respect to $E_{s}$ and $E_{a}(n=2)$ 
280-290 ${ }^{\circ} \mathrm{C}, 420^{\circ} \mathrm{C}$ and $473-490^{\circ} \mathrm{C}$, so the slowest reaction step has insignificant impacts on the distribution pattern of activation energies and vice versa. Pyrolysis of hardwood shows its major product yield at the temperature range of 250-280 ${ }^{\circ} \mathrm{C}, 314-350{ }^{\circ} \mathrm{C}$ and $437-455^{\circ} \mathrm{C}$, while pressure will vary between 50 and $75 \mathrm{~Pa}$. To predict the LBM scheme graphically, a comparative plot is drawn which precisely correlates the simulated values with the experimental curves. The solution obtained for the second order reaction has the least consistency with the experimental data, implying the hardwood pyrolysis highly favours the first order reaction or the reactions which are very near to the first order reactions.

\section{Conclusions}

The proposed scheme is successfully implemented to pyrolysis problem of biomass. It is found that the obtained solution comes within the domain of solution derived from other kinetic models. The ingression of fluid element and dispersion of fluid element on the surface of the TG plane is assumed to be part of governing DAEM equation. The fluid mass distribution is estimated to be negatively skewed on the TG surface. The velocity field is obtained at a different time scale and the fluid influx is relatively high at the beginning, which gets subdued with the time. The derived fraction of fluid mass is assumed to the part of the DAEM equation, and based on this assumption, the kinetic parameters are determined. The range of activation lies in the range of $8.44-967 \mathrm{~kJ} \cdot \mathrm{mol}^{-1}$. On the other hand, the solution is also derived for higher - order reactions to know chemical reaction pathway of hardwood pyrolysis. The activation energy for the second reaction varies in the domain of $4.22-63.24 \mathrm{~kJ} \cdot \mathrm{mol}^{-1}$. The estimated value of the pre-exponential factor for the first reaction order is estimated to $1.93 \times 1,041 \mathrm{~min}^{-1}$. The rate of reaction attains its maximum value in the temperature range of $280-290^{\circ} \mathrm{C}$. It is to be noted that the inverse formulation requires that the function must not be surjective, else the function will not exist. It implies a kinetic parameter must be unique in nature.

Apart from the evaluation of the kinetic parameters based on a hypothetical fluid medium, the percolation concept can be implemented to the simulation of the gas flow in several kinds of power generation plants.

\section{References}

BHATNAGAR, P. L. - GROSS, E. P. - KROOK, M. 1954. A model for collision processes in gases. I. Small amplitude processes in charged and neutral one-component systems. In Physical Review, vol. 94, no. 3, pp. 511-525.

BROWN, M. E. 1988. Introduction to Thermal Analysis: Techniques and Applications. London and New York (Chapman and Hall), 1988. BURNHAM, A. K. 2017. Introduction to chemical kinetics. In Global Chemical Kinetics of Fossil Fuels, pp. 25-74.

DHAUNDIYAL, A. - SINGH, S. B. 2019. Stochastic analysis of multireaction model for non-linear thermal history. In Acta Technologica Agriculturae, vol. 22, no. 3. pp. 91-97.

DHAUNDIYAL, A. - SINGH, S. B. - ATSU, D. - DHAUNDIYAL, R. 2019. Application of Monte Carlo simulation for energy modeling. In ACS Omega, vol. 4, no. 3, pp. 4984-4990.
DHAUNDIYAL, A. - SINGH, S.B. 2020 The generalisation of a multireaction model for polynomial ramping of temperature. In J Therm Anal Calorim. https://doi.org/10.1007/s10973-020-09650-7

DHAUNDIYAL, A. - TEWARI, P. 2017. Kinetic parameters for the thermal decomposition of forest waste using distributed activation energy model (DAEM). In Environmental and Climate Technologies, vol. 19, no. 1, pp. 15-32.

DHAUNDIYAL, A. - TOTH, L. 2019. Modelling of hardwood pyrolysis using the convex combination of the mass conversion points. In Journal of Energy Resources Technology, vol. 142, no. 6, pp. 061901-061910.

DHAUNDIYAL, A. - TOTH, L. 2020. Modeling of Hardwood Pyrolysis Using the Convex Combination of the Mass Conversion Points. In Journal of Energy Resources Technology, Transactions of the ASME. doi: 10.1115/1.4045458.

GALGANO, A. - DI BLASI, C. 2003. Modeling wood degradation by the unreacted-core-shrinking approximation. In Industrial and Engineering Chemistry Research, vol. 42, no. 10, pp. 2101-2111.

GIUPPONI, G. - HARTING, J.- COVENEY, P. V. 2006. Emergence of rheological properties in lattice Boltzmann simulations of gyroid mesophases. In Europhysics Letters, vol. 73, no. 4, pp. 533-539.

GUO, Z. - ZHAO, T. S. - SHI, Y. 2006. Physical symmetry, spatial accuracy, and relaxation time of the lattice Boltzmann equation for microgas flows. In Journal of Applied Physics, vol. 99, no. 7, p. 074903.

HABICH, J. - ZEISER, T. - HAGER, G. - WELLEIN, G. 2011. Performance analysis and optimization strategies for a D3Q19 lattice Boltzmann kernel on nVIDIA GPUs using CUDA. In Advances in Engineering Software, vol. 42, no. 5, pp. 266-272.

HARTING, J. - HERRMANN, H. J. - BEN-NAIM, E. 2008. Anomalous distribution functions in sheared suspensions. In Europhysics Letters, vol. 83, no. 3, p. 30001.

HECHT, M. - HARTING, J. 2010. Implementation of on-site velocity boundary conditions for D3Q19 lattice Boltzmann simulations. In Journal of Statistical Mechanics: Theory and Experiment, vol. 2010, no. 1, p. 01018.

HUANG, H. - KRAFCZYK, M. - LU, X. 2011. Forcing term in singlephase and Shan-Chen-type multiphase lattice Boltzmann models. In Physical Review E - Statistical, Nonlinear, and Soft Matter Physics, vol. 84 , no. 4.

KUTAY, M. E. - AYDILEK, A. H. - MASAD, E. 2006. Laboratory validation of lattice Boltzmann method for modeling pore-scale flow in granular materials. In Computers and Geotechnics, vol. 33, no. 8, pp. 381-395.

LI, L. - MEl, R. - KLAUSNER, J. F. 2013. Boundary conditions for thermal lattice Boltzmann equation method. In Journal of Computational Physics, vol. 237, pp. 366-395.

NEKOVEE, M. - CONEVEY, P. V. - CHEN, H. - BOGHOSIAN, B. M. 2000. Lattice-Boltzmann model for interacting amphiphilic fluids. In Physical Review, vol. E62, no. 6 B, pp. 8282-8294.

OLUOTI, K. - DODDAPANENI, T. R. K. - RICHARDS, T. E. KANAGASABAPATHI, D. 2014. Evaluation of the pyrolysis and gasification kinetics of tropical wood biomass. In BioResources, vol. 9, no. 2, pp. 2179-2190.

SERIO, M. A. - HAMBLEN, D. G. - MARKHAM, J. R. - SOLOMON, P. R. 1987. Kinetics of volatile product evolution in coal pyrolysis: Experiment and theory. In Energy and Fuels, vol. 1, no. 2, pp. 138-152.

WILK, M. - MAGDZIARZ, A. - GAJEK, M. - ZAJEMSKA, M. JAYARAMAN, K. - GOKALP, I. 2017. Combustion and kinetic parameters estimation of torrefied pine, acacia and Miscanthus giganteus using experimental and modelling techniques. In Bioresource Technology, vol. 243, pp. 304-314. 